\title{
Evidence of altered epidermal nerve fiber morphology in adults with self-injurious behavior and neurodevelopmental disorders
}

\author{
Frank J. Symons, Ph.D., \\ Department of Educational Psychology, University of Minnesota
}

Gwen Wendelschafer-Crabb, Ph.D., Department of Neurology, University of Minnesota

William Kennedy, M.D., Department of Neurology, University of Minnesota

Ronald Hardrict, M.D., Quality Care Clinic, Saint Paul, MN

Norm Dahl, Ph.D., and Department of Educational Psychology, University of Minnesota James W. Bodfish, Ph.D.

University of North Carolina at Chapel Hill

\section{Abstract}

The purpose of this preliminary study was to examine the morphology and neuropeptide density of epidermal nerve fibers quantified through skin biopsy samples from three adults with neurodevelopmental disorders and chronic self-injurious behavior (SIB) secondary to mental retardation compared with non-SIB normal IQ controls. A cross-sectional design was used with 3 $\mathrm{mm}$ punch skin biopsies collected from each participant from non-self-injurious body sites and compared with site-matched existing normal control skin samples. The study was conducted at an outpatient clinic. The primary dependent measure for the morphology analyses was the coefficient of variation $(\mathrm{CV})$ to quantify the mean gap length between epidermal nerve fibers for each subject. Visual microscopic examination and quantitative analysis of the microscopy images suggested there were morphological abnormalities (increased $\mathrm{CV}$ ) in the epidermal nerve fibers among the chronic SIB cases. Substance P (SP) fiber density was increased with 2 to 3 times as many fibers in SIB subjects as control subjects. Additional empirical work is needed to clarify the relation between sensory innervation of the skin and self-injury to improve assessment and treatment outcomes.

\section{Keywords}

cutaneous innervation; pain; self-injurious behavior

\footnotetext{
(C) 2007 International Association for the Study of Pain. Published by Elsevier B.V. All rights reserved. Corresponding author: Frank Symons, Ph.D., University of Minnesota, Minneapolis, MN 55455 USA, vm: 612.616.8697, fx: 612.624.8241, symon007@umn.edu.

Publisher's Disclaimer: This is a PDF file of an unedited manuscript that has been accepted for publication. As a service to our customers we are providing this early version of the manuscript. The manuscript will undergo copyediting, typesetting, and review of the resulting proof before it is published in its final citable form. Please note that during the production process errors may be discovered which could affect the content, and all legal disclaimers that apply to the journal pertain.
} 
Self-injurious behavior (SIB) is among the most destructive forms of behavior disorder observed in individuals with neurodevelopmental disorders (Schroeder, Oster-Granite, \& Thompson, 2002). In almost all cases of SIB the pathogenesis is unknown and the underlying pathophysiology is only partly understood. Clinical observation suggests that some individuals with developmental disorders who persistently self-injure appear to be insensitive to actions that would normally be painful to others, yet the role of behavioral and neurological factors related to pain in general and nociception in particular in individuals with neurodevelopmental disorders and self-injury has received little systematic study (Barrera, 1994).

Chronic SIB has been shown to be associated with behavioral (Breau, et al., 2003; Oberlander, et al., 2003), physiological (Symons, Sutton, \& Bodfish, 2001), and biochemical (Symons, et al., 2003) correlates consistent with altered nociception suggesting mediation by abnormal pain sensitivity in at least a subset of cases. Altered pain sensitivity is associated with morphological changes in cutaneous epidermal nerve fibers (ENFs) in a variety of clinical conditions (Kennedy et al. 2005; Kennedy, Wendelschafer-Crabb, \& Johnson, 1996; McCarthy et al., 1995). Although the exact mechanisms regulating altered pain sensitivity associated with structural changes in sensory afferents are unclear, preclinical models suggest that neuropathic pain resulting from lesions in the peripheral nervous system is mediated by a number of mechanisms including sensitization of spinal cord dorsal horn neurons (Woolf, 2004), and inflammatory and immune peptides (Moalem \& Tracey, 2006).

Epidermal nerve fibers are free nerve endings widespread in the epidermal layers of human skin and can be visualized by using the pan neuronal maker protein gene product 9.5 (PGP 9.5) (Kennedy \& Wendelschafer-Crabb, 1993). Nolano et al (2000) used immunohistochemistry to visualize nerve fibers from skin biopsies from a single case with a confirmed sensory disorder (congenital insensitivity to pain with anhidrosis [CIPA]) to examine, in part, the morphological correlates underlying the sensory abnormalities. The young girl presented with mental retardation and insensitivity to pain in addition to having a long history of chronic SIB (finger/hand biting). Myelinated nerves appeared to be intact but immunohistochemistry of skin samples showed an almost complete lack of unmyelinated nerve fibers in the epidermis. Although the specific relation between the child's self-injury and lack of epidermal innervation was not clear, this was the first reported demonstration showing the morphological basis for analgesia in CIPA.

Pain abnormalities and nociceptive deficits in patients with borderline personality disorder (BPD) and SIB have been reported with many patients reporting not feeling pain during selfinjury (Kemperman, 1997), demonstrating altered thermal responsivity to noxious stimuli (Russ et al., 1993), and attenuated pain perception (Schmahl, et al., 2004). No work of a similar nature has been conducted among nonverbal individuals with mental retardation and related neurodevelopmental disorders and chronic self-injury. The purpose of this preliminary study was to examine the morphology of epidermal nerve fibers quantified through skin biopsy samples from three individuals with neurodevelopmental disorders and chronic SIB compared with a non-SIB normal control extant database.

\section{Methods}

\section{Subjects}

Three adults (2 male) with severe (1) and profound (2) cognitive impairment and associated neurodevelopmental disability and histories of chronic tissue damaging self-injury participated in this study. Each individual had (1) a functional diagnosis of severe/profound mental retardation/developmental disability (based on DSM IV criteria and standard intellectual and adaptive behavioral test results as documented through existing chart 
review); (2) exhibited SIB either hourly or at least daily in bouts or discrete episodes (based on parent or teacher report and corroborated through direct observation); (3) chronic SIB (present for at least that last 12 months) (based on parent or teacher report); (4) received treatment for SIB that was currently stable (not planned on being changed in the next month); and (5) parent or guardian informed consent. Normal values were obtained from an extant cohort of healthy adult subjects $(n=45)$

The mean age of the three participants was 32 (Range $=20-40)$. SIB had been in the behavioral repertoire of each individual since early childhood. The primary SIB location was the front and side of the forehead and the back and side of the hands. Locations appeared to be symmetrical. The primary SIB form was forceful contact of closed fist with the head or face and biting. Each SIB site was associated with surface trauma marked by breaks in the skin, tissue rupture, and scarring. Each individual was in overall good health with no serious accompanying chronic health impairments considered to be painful (e.g., chronic reflux or otitis media as determined by subjects' physician record review and/or examination if necessary) or specific syndromes associated with SIB (e.g., Lesch-Nyan syndrome, Cornelia de Lange syndrome).

\section{Skin Biopsy}

Following IRB approval and informed consent from the University of Minnesota Committee for the Protection of Human Subjects, single punch skin biopsies were obtained from a normative site (i.e., no skin damage, no SIB) to compare directly with the same normative samples from age-matched, body-site matched control adults without developmental disability. In all cases, (SIB, control) the body site was the inside forearm (medial of midline where the lower $2 / 3$ meets upper $1 / 3$ over FCR). Body sites were anesthetized by intradermal injection of $1 \%$ xylocaine or the application of a topical anesthetic and the biopsy was made with a $3 \mathrm{~mm}$ punch tool (Aucpunch; Acuderm; Fort Lauderdale, FL).

Biopsies were fixed in Zamboni's solution, cryoprotected, and sectioned with a freezing sliding microtome (Leica, Nussloch, Germany). Diluent and washing solutions were $1 \%$ normal donkey serum (Jackson ImmunoResearch, West Grove, PA) in 0.1 M PBS with 0.3\% Triton X-100 (Sigma, St. Louis, MO). Floating sections were blocked with 5\% normal donkey serum in the diluent solution. Nerve and tissue antigens were localized using primary antibodies to protein gene product (PGP) 9.5 (1:800; Biogenesis, Kingston, NH), substance P (SP) (1:1000; Diasorin, Stillwater, MN), calcitonin gene-related peptide (CGRP) (1:1000; Diasorin, Stillwater, MN) and type IV collagen (1/800; Chemicon, Temecula, CA), with each diluted in PBS-Triton X-100-NGS. Nonimmune serum was used with negative controls. Secondary antibodies labeled with cyanine dye fluorophores 2 or 3 (Jackson ImmunoResearch), were used to locate two antigens in each section. After immunohistological processing, sections were adhered to coverslips with agar, dehydrated via an alcohol series, cleared with methyl salicylate, and mounted in DPX (Fluka BioChemika, Ronkonkoma, NY).

Sections were evaluated visually with a Nikon Microphot-SA fluorescent microscope (Lake Success, NY) for qualitative assessment of nerve morphology and neuropeptide density. Calcitonin gene related peptide (CGRP) and substance $\mathrm{P}$ (SP) positive fibers in the subepidermal neural complex, superior to the papillary capillary complex, were counted as they approached, in a vertical orientation, the epidermal basement membrane. Neuropeptidepositive nerve fibers within or underlying the capillary complex were not counted.

Thick sections were confocally imaged (CARV non-laser confocal microscope imaging system, Atto Bioscience Inc. Rockville, MD) and then epidermal nerve fibers traced with Neurolucida software (MicroBrightField, Colchester, VT.) according to established counting 
criteria and reported as number of epidermal nerve fibers per $\mathrm{mm}$ in a $50 \mu \mathrm{m}$ thick section (Kennedy et al. 1996). Gaps between nerve fibers were determined based the x,y,z coordinates of ENF/basement membrane intersections across the full length of a section $(3 \mathrm{~mm})$ using the three dimensional Neurolucida tracing of multiple montaged confocal images The primary dependent measure for the analyses of distribution was the coefficient of variation $(\mathrm{CV})$ of the mean gap length between epidermal nerve fibers for each subject.

\section{Results}

Visual microscopic examination and qualitative analyses of the microscopy images suggested there were morphological abnormalities characterized as 'sprouting' or 'tufting' abnormalities in the epidermal nerve fibers among the chronic SIB cases (similar with that observed in some cases of small fiber peripheral neuropathies). Variations in ENF distribution resulting from the tufting and clustering of nerve fibers in the SIB cases were clearly observed. A confocal image of a normal control forearm skin biopsy (Fig. 1a) shows the uniform distribution and normal density of epidermal nerve fibers. Images from a representative SIB case (Fig. 1b,c) display the altered distribution of the epidermal nerve fibers. Epidermal nerve fiber density for the three subjects was calculated as 37.2, 27.6, and 27.6 epidermal nerve fibers $/ \mathrm{mm}$ in a $50 \mu \mathrm{m}$ thick section. Normal epidermal nerve fiber density for forearm is 36.4 with a $95 \%$ cutoff of 17.8 . No significant nerve density changes were observed. Quantitative analyses of gaps between ENFs revealed significant distributional abnormalities in the spacing of the epidermal nerve fibers in the SIB group as indexed by calculating the coefficient of variation (CV). The chronic SIB cases had a greater $\mathrm{CV}(\mathrm{M}=108.58)$ relative to controls $(\mathrm{M}=79.67)$ in which SIB $\mathrm{CV}$ values exceeded the 95\% cutoff of the control distribution (i.e., more variable and irregular spacing relative to controls). Analysis of peptides showed normal levels of CGRP but SP levels were elevated in the chronic SIB cases. SP fiber density was increased with 2 to 3 times as many fibers in SIB subjects as control subjects (Fig. 1d). VIP was not present in the SNP of either SIB or control subjects (Table 1). Links between the ENF distribution findings and the peptide content findings for these cases were also examined. As evident in Fig 1 (b) it appeared that there was some pathology in the way the innervation approached the epidermis. In particular, the entire stratum of fibers that distributed parallel to, and immediately subjacent to the epidermis, was virtually absent in some of the SIB cases and much of the innervation appeared to be from relatively deep fibers.

\section{Discussion}

We hypothesized that there may be a SIB subtype associated with a specific pattern of morphological and neurochemical markers characterized by altered function or structure of the sensory nerve fibers. We found preliminary evidence for altered morphology and neurochemistry in three adults with chronic SIB. The results from this novel immunohistological analysis of skin in individuals with severe neurological impairment and SIB do not definitively confirm or refute a SIB subtype model, but do suggest that there may be significant differences in the morphology of nerve fibers in at least a portion of chronic SIB cases. The significance of these findings is underscored by the fact that the biopsy was sampled at a non-SIB site with no history of tissue damage.

In the absence of a non-SIB neurodevelopmental control group and the small sample size, it is not clear whether the findings are specific to SIB or the underlying neurodevelopmental disorder. Because invasive work on vulnerable populations with no direct benefit is extremely difficult to perform ethically, we decided on a conservative approach by making initial normative comparisons. Each individual was nonverbal and we were unable to perform standard electrophysiological or psychophysical tests of sensory function that rely 
on self-report to corroborate clinical impressions of altered sensation. Thus, we cannot conclude definitively that the SIB cases had altered (i.e., increased/decreased) pain sensitivity.

The clinical presentation of chronic, high frequency, tissue-damaging acts leads to two broad sets of questions. First, what is the experience of pain like in persons who exhibit SIB? The difficulty with this question is that the majority of individuals in question have significant cognitive and language deficits that do not permit them to describe their pain experience in a conventional manner (Defrin et al., 2004). They cannot tell us (a) if they are in pain or experiencing some form of unusual and perhaps discomforting sensation and if so whether they engage in SIB in reaction to this uncomfortable sensory state, (b) if they are not in pain and do not feel pain or unusual sensory experiences following SIB, or (c) if they are not in pain but experience some type of desirable (for them) sensory experience following SIB. The second set of questions concern the physiological mechanisms involved in pain perception. Because each of the subjective scenarios just described are plausible but not easily or readily testable in persons with severe cognitive impairment using conventional means (i.e., self-report), our aim was to examine some of the structural and chemical features of ENFs that are known to be linked to peripheral sensory perception and experience. As this was a case series we obviously cannot address each scenario definitively. We can, however, begin by considering how the pattern of ENF results obtained for the SIB cases differs from normal and converges with what has been shown for other pathological conditions associated with altered sensory and/or pain perception/processing.

The observed ENF distribution variability (CV), characterized by significantly more gaps between ENFs in each SIB case relative to the normal controls, raises the possibility that a once normal distribution of ENFs degenerated to the point of obvious ENF gaps in these cases. Epidermal nerve fibers have been demonstrated to degenerate in a variety of painful neuropathic conditions (Kennedy et al. 2005). Altered or disrupted pain sensitivity is influenced, in part, by such morphological changes in cutaneous ENFs under normal and clinical pain conditions (Kennedy, et al., 1996). This raises the possibility that functionally, persons with SIB with abnormal ENF innervation could experience atypical sensory experiences characterized by a relative insensitivity to pain. If so, pain insensitivity could plausibly either lead to or maintain persistent self-injury due at least in part to a failure of the typical pain-related feedback that would make self-injurious acts aversive. The importance of this hypothesis is that it would provide a putative biological marker (abnormal ENF innervation) for prospective longitudinal studies of the early development of SIB in at-risk populations.

Conversely, the morphological characteristics found in the present SIB cases could also be interpreted as consistent with increased pain sensitivity as previous work has found that altered ENF distribution and innervation is associated with clinical conditions with altered pain experience (Albrecht et al. 2006; Kennedy, et al., 1996). This leads to two plausible functional consequences for persons with SIB. First, abnormal ENF morphology could lead to abnormal sensory experiences including pain and SIB may develop in response to this aversive sensory state. Second, if as anecdotal evidence suggests persons with SIB are indeed insensitive to pain, then this pattern of altered ENF morphology may have more to do with how normal/abnormal pain perception evolves than with altered pain mechanisms per se. The additional finding that SP levels were higher in SIB cases is suggestive that a balance of peptidergic and nonpeptidergic innervation may be required to establish normal tactile perception and, consequently, the comparative contrasting basis that is necessary for the development of a notion of pain. 
The developmental consequences of a congenital absence of normal nonpeptidergic peripheral innervation are largely unknown. The purported lack of normal perception might also contribute to failure to develop proper cognitive mechanisms and thus be a causative factor in the development not only of SIB but also of mental retardation more generally. This is relevant because cognitive impairment in general and mental retardation syndromes in particular appear to be the strongest risk factors for the development of SIB. This is a novel conceptualization of etiologic factors involved in cognitive development (and conversely mental retardation) that could conceivably direct a search for genetic factors known to be involved in the development of peripheral pain mechanisms for application in association or linkage studies designed to identify genetic mechanisms of mental retardation and/or SIB. It is not well understood whether and how a failure in the normative development in one system/domain (i.e., sensory/perceptual) may have consequences for the normative development in another (i.e., cognitive). Emerging work on the neuronal circuitry for the development and modulation of pain perception (Dan \& Mooney, 2006) may be particularly relevant to prospective studies designed to understand sensory function in the context of congenital neurologic impairment.

The observation of relatively deep-fiber innervation could be relevant for the pathophysiology of SIB given previous study findings showing that peptidergic and nonpeptidergic epidermal innervation is segregated spatially (Zylka et al., 2005). Deeper fiber strata have been shown to contain a high proportion of the peptidergic innervation and this is consistent with the peptide findings for the SIB cases which showed a 2-3 fold increase in SP fiber density. This is a preliminary observation, however, and in terms of any possible pathology in the way the innervation approached the epidermis, care must be taken in making broad judgments with regard to morphology based on single images with few participants. In the case of the sub-epidermal neural plexus (SNP) in human skin biopsies, a great deal of variation is seen in normal biopsies, from dense networks that are intimately opposed to the epidermal basement membrane to more sparse plexuses located between the capillary plexus and the epidermal basement membrane. Frequently the plexus has a horizontal aspect, occasionally more prominent vertical fiber bundles are observed. Single nerve fiber terminals arise from the SNP and pass through the basement membrane to innervate the epidermis. The appearance of the SNP can be affected by local morphology such as nearby sweat ducts, hair follicles or skin contours. Although the larger nerve bundles passing through the dermis include neuropeptide positive nerve fibers and they were the source of the neuropeptide fibers that we reported in the SNP, careful review of sections did not reliably find consistent uniform overt abnormalities in the SNP. Increasing the sample size would permit a more conclusive analysis to resolve the issue.

It is also plausible that neurotrophic factors could play an important role in the pathophysiology underlying the observed ENF abnormalities. Neurotrophic factors (e.g. BDNF, NGF) are implicated in a variety of neurodegenerative disorders that are associated with severe cognitive impairments (e.g. Alzheimer's disease; Elliot \& Ginzburg, 2006). There is an established link between neurotrophic factors and differential cutaneous innervation (Rice, et al., 1998) that has been increasingly explored in relation to pathological conditions through animal models (Yamaoka, et al., 2007) and human cutaneous disorders (Dou, et al., 2006) suggesting that any comprehensive examination of the developmental course of ENF morphology should include close analyses of neurotrophic factors.

To begin studying peripheral factors in relation to repeated self-injury, previous work examined the specific body site locations of self-injury (Symons \& Thompson, 1997; Symons, et al., 1993; Symons, et al., 2003) and physiological correlates at those sites (Symons, et al., 2001). Further work appears warranted to (a) conduct confirmatory tests of differences in the morphology of nerve fibers in relation to SIB, (b) determine the regulatory 
processes underlying early development of epidermal nerve fibers and later plasticity, and (c) understand the functional significance of morphological differences among individuals with neurodevelopmental disorders.

\section{Acknowledgments}

Supported, in part, by a McKnight Land-Grant Professorship (Symons) and NIH Grant No. HD44763. Our thanks to dedicated parents and their children.

\section{References}

Albrecht PJ, Hines S, Eisenberg E, Pud D, Finaly DR, Connolly MK, Pare M, Davar G, Rice FL. Pathologic alterations of cutaneous innervation and vasculature in affected limbs from patients with complex regional pain syndrome. Pain. 2006; 120:244-266. [PubMed: 16427199]

Barrera FJ, Teodoro JM, Selmeci T, Madappuli A. Self-injury, pain, and the endorphin theory. J Dev Phys Disab. 1994; 6:169-192.

Breau L, Boll P, McKay A, Symons FJ, et al. Pain and self-injurious behavior in neurologically impaired children. J Ped. 2003; 142:498-503.

Dan Y, Mooney RD. Sensory systems. Curr Op Neurobio. 2006; 16:359-362.

Dou YC, Hagstromer L, Emtestam L, Johannson O. Increased nerve growth factor and its receptors in atopic dermatitis: an immunohistochemical study. Arch Dermatol Res. 2006; 296:31-37. [PubMed: 16586073]

Elliot E, Ginzburg I. The role of neurotrophins and insulin on tau pathology in Alzheimer's disease. Rev Neurosci. 2006; 17:635-642. [PubMed: 17283608]

Iwata BA, Pace GM, Kissel RC, Nau PA, Farber JM. The self-injury trauma (SIT) scale: A method for quantifying surface tissue damage caused by self-injurious behavior. J Appl Beh Analy. 1990; 23:99-110.

Kennedy WR, Wendelschafer-Crabb G. The innervation of human epidermis. J Neurol Science. 1993; 115:184-190.

Kennedy WR, Wedelnschafer-Crabb G, Johnson T. Quantification of epidermal nerves in diabetic neuropathy. Neurol. 1996; 47:1042-1048.

Kennedy, WR.; Wendelschafer-Crabb, G.; Polydefkis, M.; McArthur, J. Pathology and Quantitation of cutaneous nerves. In: Dyck, PJ.; Thomas, PK., editors. Peripheral Neuropathy. 4th ed.. Philadelphia: Saunders; 2005. p. 869-896.

McCarthy BG, Hsieh ST, Stocks A, Hauer P, Macko C, Cornblath DR, Griffin JW, McArthur JC. Cutaneous innervation in sensory neuropathies: Evaluation by skin biopsies. Neurol. 1995; 45:1848-1855.

Moalem G, Tracey DJ. Immune and inflammatory mechanisms in neuropathic pain. Brain Res Rev. 2006; 51:240-264. [PubMed: 16388853]

Newell KM, Challis JH, Boros RL, Bodfish JW. Further evidence on the dynamics of self-injurious behaviors: Impact forces and limb motions. Am J Mental Retard. 2002; 107:60-68.

Nichols ML, Allen BJ, Rogers SD, et al. Transmission of chronic nociception by the spinal neurons expressing the substance P receptor. Science. 1999; 286:1558-1561. [PubMed: 10567262]

Nolano M, Crisci C, Santoro L, Barbieri F, Casale R, et al. Absent innervation of skin and sweat glands in congenital insensitivity to pain with anhidrosis. Clin Neurophys. 2000; 111:1596-1601.

Oberlander, TF.; Symons, FJ.; Van Donge, K.; Abu-Saad, HH. Pain in individuals with developmental disabilities: Challenges for the future. In: Dostrovsky, O.; Carr, DB.; Klotzenburg, M., editors. Progress in pain research and management. Seattle: IASP Press; 2003. p. 705-722.

Rice FL, Albers KM, Davis BM, Silos-Santiago I, Wilkinson GA, LeMaster AM, Ernfors P, Smeyne RJ, Aldskogius H, Phillips HS, Barbacid M, DeChiara TM, Yancopoulos GD, Dunne CE, Fundin BT. Differential dependency of unmyelinated and A delta epidermal and upper dermal innervation on neurotrophics, trk receptors, and p75LNGFR. Dev Biol. 1998; 198:57-81. [PubMed: 9640332]

Schmahl C, Greffrath W, Baumgartner U, Schlereth T, Magerl W, et al. Differential nocicepetive deficits in patients with borderline personality disorders and self-injurious behavior: laser-evoked 
potentials, spatial discrimination of noxious stimuli, and pain ratings. Pain. 2004; 110:470-479. [PubMed: 15275800]

Symons FJ, Butler MG, Sanders MD, Feurer ID, Thompson T. Self-injurious behavior and PraderWilli syndrome: Behavioral forms and body locations. Am J Mental Retard. 1999; 104:260-269. (1999).

Symons FJ, Clark RD, Hatton DD, Skinner M, Bailey DB. Self-injurious behavior in young boys with fragile X syndrome. Am J Med Gen. 2003; 118:115-121.

Symons FJ, Sutton KA, Bodfish JW. A preliminary study of altered skin temperature at body sites associated with self-injurious behavior in adults with developmental disabilities. Am J Mental Retard. 2001; 106:336-343.

Symons FJ, Sutton KA, Walker C, Bodfish JW. Altered diurnal pattern of salivary substance P in adults with developmental disabilities and chronic self-injury. Am J Mental Retard. 2003; 108:1318.

Symons FJ, Thompson T. Self-injurious behavior and body site preference. J Int Dis Res. 1997; 41:456-468.

Woolf CJ. The pathophysiology of peripheral neuropathic pain - abnormal peripheral input and abnormal central processing. Act Neurochir Suppl. (Wien). 1993; 58:125-130.

Woolf CJ. Dissecting out mechanisms responsible for peripheral neuropathic pain: implications for diagnosis and therapy. Life Sci. 2004; 74:2605-2610. [PubMed: 15041442]

Yamaoka J, Di ZH, Sun W, Kawana S. Changes in cutaneous sensory nerve fibers induced by skin scratching in mice. J Dermatol Sci. 2007; 46:41-51. [PubMed: 17239567]

Zylka MJ, Rice FL, Anderson DJ. Topographically distinct epidermal nociceptive circuits revealed by axonal tracers targeted to Mrgprd. Nueron. 2005; 45:17-25. 

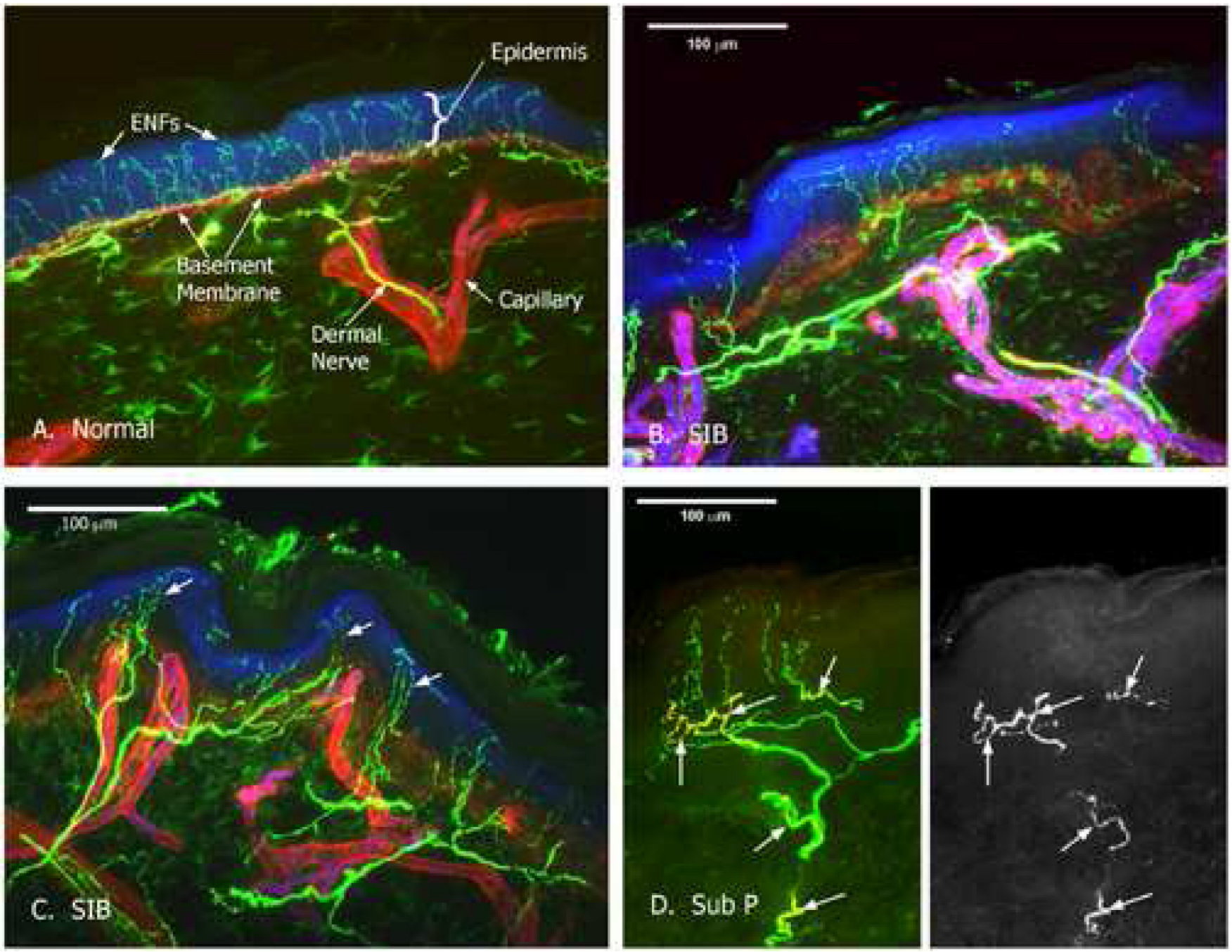

Figure 1.

Confocal images of human skin. Nerves appear green, basement membrane surrounding capillaries and the dermal-epidermal (d/e) boundary appears red. The epidermis is blue. (a) Control skin sample with normal innervation pattern. Epidermal nerve fibers (ENFs) penetrate the $\mathrm{d} / \mathrm{e}$ basement membrane in a uniform pattern; (b) Representative skin sample from a non-involved site of a self-injurious behavior (SIB) case from same plane of section as control demonstrating intact subepidermal stratum; (c) Representative skin sample from non-involved site of a SIB case demonstrating tufting and clustering; (d) SIB case substance $\mathrm{P}$ immunolabeling. 
